\title{
Adaptive Robust Sliding Mode Vibration Control of a Flexible Beam Using Piezoceramic Sensor and Actuator: An Experimental Study
}

\author{
Ruo Lin Wang, ${ }^{1}$ H. Gu, ${ }^{2}$ and G. Song ${ }^{2,3}$ \\ ${ }^{1}$ School of Civil Engineering, Wuhan University, Wuhan, Hubei 430072, China \\ ${ }^{2}$ Department of Mechanical Engineering, University of Houston, Houston, TX 77204, USA \\ ${ }^{3}$ School of Civil Engineering, Dalian University of Technology, Dalian, Liaoning 116024, China \\ Correspondence should be addressed to G. Song; gsong@uh.edu
}

Received 28 September 2013; Revised 23 December 2013; Accepted 8 January 2014; Published 26 May 2014

Academic Editor: ShengJun Wen

Copyright ( 2014 Ruo Lin Wang et al. This is an open access article distributed under the Creative Commons Attribution License, which permits unrestricted use, distribution, and reproduction in any medium, provided the original work is properly cited.

\begin{abstract}
This paper presents an experimental study of an adaptive robust sliding mode control scheme based on the Lyapunov's direct method for active vibration control of a flexible beam using PZT (lead zirconate titanate) sensor and actuator. PZT, a type of piezoceramic material, has the advantages of high reliability, high bandwidth, and solid state actuation and is adopted here in forms of surface-bond patches for vibration control. Two adaptive robust sliding mode controllers for vibration suppression are designed: one uses a discontinuous bang-bang robust compensator and the other uses a smooth compensator with a hyperbolic tangent function. Both controllers guarantee asymptotic stability, as proved by the Lyapunov's direct method. Experimental results verified the effectiveness and the robustness of both adaptive sliding mode controllers. However, from the experimental results, the bang-bang robust compensator causes small-magnitude chattering because of the discontinuous switching actions. With the smooth compensator, vibration is quickly suppressed and no chattering is induced. Furthermore, the robustness of the controllers is successfully demonstrated with ensured effectiveness in vibration control when masses are added to the flexible beam.
\end{abstract}

\section{Introduction}

Model inaccuracies or uncertainty can have adverse effects on control systems. The adaptive control and the sliding mode control are two major nonlinear control methods to deal with model uncertainties. The typical structure of a sliding mode controller is composed of estimated terms of the system, to the best of knowledge, and a robust compensator, which deals with model uncertainty and disturbance to ensure stability. The robust compensator usually consists of an upper bound of the system uncertainty with a nonsmooth switching function, such as a sign function or a saturation function. This robust compensator is very important in the sliding mode control design since the robust compensator will make the system trajectory converge toward the sliding surface in a finite time and remain on the sliding surface under system uncertainties and external disturbances. The asymptotic stability is achieved by the proper design of the robust compensator in the sliding mode control. The Lyapunov's direct method is often used to assist the design and to provide a stability proof.

Song and Mukherjee [1] presented a comparative study of two nonsmooth time-invariant robust compensators (the bang-bang compensator and the saturation compensator) with a smooth time-varying compensator. The comparative study reveals the superiority of the smooth, time-varying compensator over the nonsmooth robust compensator. Many scholars have studied and developed different kinds of sliding mode control schemes. Park et al. [2] proposed a twostage sliding mode controller for vibration suppression of a flexible pointing system. Yu et al. [3] proposed a fuzzy sliding mode control strategy which consists of fuzzily amalgamated sliding mode controls of the system linearized around a set of operating points. There are also many other sliding mode control schemes. An adaptive sliding mode control takes advantage of aspects from both the adaptive control and the 
sliding mode control schemes. The adaptive control approach is suitable for control of the dynamic systems with constant or slowly varying uncertain parameters. The parameters of the adaptive controller are updated during operation based on the measured performance. The adaptive control law is designed to guarantee the asymptotic stability for the control system under disturbances.

With merits of both the adaptive control and the sliding mode control schemes, adaptive sliding mode control can achieve satisfactory control performance with asymptotic stability when the system is subject to model uncertainties and disturbances. Due to these advantages, the adaptive sliding mode control has been studied extensively for the control of dynamic systems with uncertainties and disturbances. Yao and Tomizuka [4] proposed a systematic way to combine the adaptive control design technique and the sliding mode control methodology for trajectory tracking control of robot manipulators in the presence of parametric uncertainties and external disturbance. Wang et al. [5] proposed an indirect, adaptive fuzzy sliding mode control scheme for a class of nonlinear systems by using the concept of sliding mode control design and Lyapunov synthesis approach. Simulation studies have shown that the adaptive design of fuzzy sliding mode controller performs very well in the presence of an unknown disturbance. Song et al. [6] proposed an integrated, adaptive-robust control methodology for control of nonlinear uncertain systems. The new approach can take advantage of both adaptive and robust control methods and it offers to handle various uncertainties, reject external disturbance, and guarantee global asymptotic stability with desired transient response. Song et al. [7] proposed an integrand, adaptive-robust approach along with a smooth, adaptive robust friction compensation strategy for tracking the control of uncertain robot manipulators with joint stick-slip friction. Wai [8] proposed an adaptive sliding mode control system to control the position of an induction servomotor drive. In the adaptive sliding mode control system, an adaptive algorithm is utilized to estimate the bound of uncertainties. Simulated and experimental results show that the dynamic behaviors of the proposed control systems are robust with regard to uncertainties. Lin and Chen [9] developed a new strategy called adaptive fuzzy sliding mode control (AFSMC). The parameters of the membership functions in the AFSMC are changed according to some adaptive algorithm for the purpose of controlling the system states to converge to a user-defined sliding surface and then slide along it. The sliding mode control can achieve satisfactory vibration control performance since the vibration control system is usually a nonlinear system with model uncertainty and external disturbance. Fei and Ding [10] proposed a radial basis function neural network adaptive sliding mode controller to track a microelectromechanical system triaxial gyroscope by integrating the adaptive control, neural network control, and sliding mode control. Switching function and sliding mode controller were used as the input and output of the radial basis function of neutral network to deal with nonlinearity with an online learning ability. Zhang and Han [11] applied robust sliding mode $\mathrm{H}_{\infty}$ control using timevarying delayed states for an offshore steel jacket platform with self-exciting nonlinear wave force, external disturbance, and parametric uncertainties. Zhang et al. [12] proposed a new variable structure sliding mode control strategy with a combined approaching law for fast tool servo to control cutting force in diamond-cutting microstructured surfaces. Li et al. [13] proposed an adaptive sliding mode control via Takagi-Sugeno (T-S) fuzzy approach to control suspension system. The T-S fuzzy control was used to describe the original nonlinear system for the control design via a sector nonlinearity approach. A sufficient condition, which could be converted to convex optimization problem, is proposed for asymptotical stability of the designing sliding motion.

Piezoelectric materials are commonly used in the vibration control as actuators and sensors. Piezoelectric material is an innovative smart material with the advantages of quick response, low-power cost, and easy implementation. During the past years, piezoelectric material has been successfully applied in different kinds of active or passive vibration control methods such as PPF control $[14,15]$, fuzzy model reference control [16], vibration control for robotic links [17, 18], and passive vibration control with shunted piezoelectric material [19]. Fei et al. [20] compares the uses of PZT-based optimal PID, strain rate feedback (SRF), and PPF control schemes on the vibration control of a cantilever beam. Piezoelectric material-based sliding mode control provides an innovative and effective strategy for the vibration control of systems with model inaccuracy and uncertainties. Choi et al. [21] developed an active vibration control of a hybrid smart structure consisting of a piezoelectric film actuator and an electrorheological fluid actuator. A neuro-sliding mode controller incorporating neural networks with the concept of sliding mode control is formulated for a piezoelectric film actuator. Actuators and sensors made of piezoelectric materials have the merits of low weight and distributed nature. Jha and Inman [22] utilized piezoelectric actuators and sensors in the vibration suppression of gossamer structures. In order to achieve good vibration reduction performances under model uncertainty and external disturbance, a sliding mode controller and observer are designed. The simulation studies show that the piezoelectric actuators and sensors are suitable for vibration suppression of an inflatable torus. Choi and Kim [23] proposed a new discrete-time, fuzzy sliding mode controller and applied it to vibration control of a smart structure with a piezofilm actuator. Experimental results of transient, forced, and random vibration of smart structures demonstrate the effectiveness of the proposed method. Recent studies by other researchers have refined sliding mode control methods for use in micro/nanoactuators [24-26]. Hu and Zhu [27] applied a sliding mode control with observer to reduce the vibration of a flexible structure with a piezoelectrical actuator and a strain gauge sensor. A state space dynamic system was derived by using a finite element method and the experimental modal test. The controller's sliding surface was determined by using optimization method that minimized the cost function of the states. There are also many other schemes of sliding mode control applied to vibration control by using piezoelectric material.

Many simulation studies have been performed to make contributions to the theoretical study of the adaptive sliding 
mode control. Some recent studies, for example, have investigated self-tuning laws especially for uncertain nonlinear systems [28] and advanced observer-based robust control of piezoelectric structures [29]. However, not enough experimental study of the adaptive sliding mode control has been conducted. The adaptive sliding mode controller is designed to handle the system uncertainties and disturbances, and it is more important to conduct the experimental study to verify the robustness of the proposed controller under real systems with uncertainties and disturbances than under the simulated systems. Experimental verification of adaptive sliding mode controller is an important issue for the study of the adaptive sliding mode controller. The real-time experimental results are more convincing in verifying and proving the robustness of an adaptive sliding mode control than the simulation results. In this paper, experimental comparative study of two different adaptive robust sliding mode controllers is conducted. An adaptive robust sliding mode control scheme, based on the Lyapunov's direct method, is proposed for active vibration control of a flexible beam using PZT (lead zirconate titanate) sensors and actuators. PZT, a commonly used piezoelectric ceramic material, is adopted in this research, and surface-bond PZT patches were used for vibration control. Two adaptive robust sliding mode controllers for vibration suppression were designed: one adaptive sliding mode controller uses a discontinuous bang-bang robust compensator and the other uses a compensator with a smooth, hyperbolic tangent function. The Lyapunov's direct method is used to prove the asymptotic stability of both controllers. The proposed adaptive sliding mode controller reduces the conservatism of the sliding mode controller. The asymptotic stability of the proposed control system was proved by using the Lyapunov's direct method. Experiments have been implemented on a flexible beam with PZT actuators and sensors. Experimental results show that both controllers successfully suppress vibrations of the flexible beam in an efficient way. However, the bang-bang robust compensator causes smallmagnitude chattering because of the discontinuous switching actions. With the smooth compensator, vibration is quickly suppressed and no chattering is induced. Furthermore, the robustness of the controllers is successfully tested by adding mass to the flexible beam. Multimode vibration control of the beam with mass added is also conducted to verify the effectiveness and robustness of the proposed adaptive sliding mode control in vibration control.

\section{Experimental Setup}

A flexible aluminum beam in a cantilevered configuration (Figure 1) is used as the experimental object to test the effectiveness of the proposed vibration suppression method. The property of the beam is shown in Table 1. PZT type piezoelectric patches are used as the smart sensor and actuator. One relatively large PZT patch is surface-bonded on each side of the flexible aluminum beam near its cantilevered root. These two patches are used as actuators to excite and to enable active vibration control of the beam. One smaller PZT patch is surface-bonded on one side of the beam, also near its
TABLE 1: Beam properties.

\begin{tabular}{lccc}
\hline Symbol & Quantity & Unit & Value \\
\hline$L$ & Beam length & $\mathrm{mm}$ & 736.5 \\
$w_{b}$ & Beam width & $\mathrm{mm}$ & 53.1 \\
$t_{b}$ & Beam thickness & $\mathrm{mm}$ & 1 \\
$\rho_{b}$ & Beam density & $\mathrm{kg} / \mathrm{m}^{3}$ & 2690 \\
$E$ & Modulus of elasticity & $\mathrm{N} / \mathrm{m}^{2}$ & $7.03 \times 10^{10}$ \\
\hline
\end{tabular}

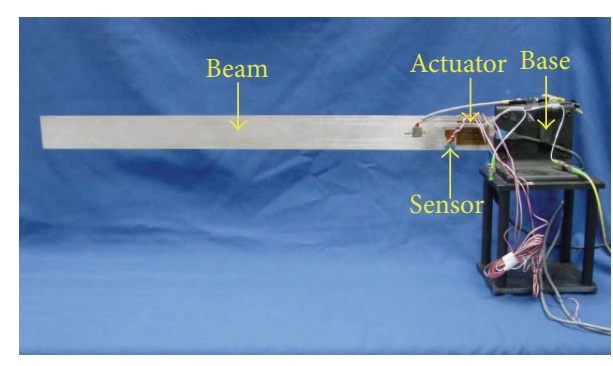

Figure 1: The experimental setup.

cantilevered root. This smaller PTZ patch acts as sensor for the feedback of the signals in the active control algorithms. Physical position of PZT actuators and sensors is shown in Figure 2. Both the PZT actuators and sensor are installed near the cantilevered root of the beam since this location has the highest strain energy along the length of the beam. This configuration of actuator and sensor placement results in the most effective actuation and sensing for vibration control. The properties of the PZT patches are shown in Table 2.

A PZT actuator has some nonlinear characteristics, such as hysteresis. Hysteresis should be compensated for accurate positioning for position regulation or tracking tasks. The hysteresis width of PZT is about $10 \%$ of its stroke, and normally PZT actuator is not classified as a "high" nonlinear actuator. In addition, the hysteresis width reduces with the increasing frequency. Therefore, for active vibration control, it is a common practice that the hysteresis is not considered in such an application. The nonlinear characteristics of PZT transducers are not considered in this paper.

Experiments have shown that the dominant mode of the flexible beam is its first mode and is the major concern for vibration suppression (details can be found in Section 5). Since the first mode is dominant in the experiment, higher modes are truncated for simplification. Bu et al. [30] derived the model for a flexible smart beam with piezoceramic actuators and strain gauges. When the first mode is dominant in the analysis (i.e., only the first mode is considered), model between PZT actuator voltage and strain sensor can be represented as a simplified linear second order system, which validates the practice of not considering the nonlinear characteristics of PZT transducers in vibration control problems. The PZT sensor output represents the strain of the sensor location. Therefore, the PZT sensor can be applied as strain gauge for control purposes. In the control design, a simplified second order model is used as the structure of the model. However, the model parameters are assumed unknown. The design of the controller is not dependent on the parameters 
TABle 2: Properties of PZT patches used on the beam.

\begin{tabular}{lcccc}
\hline Symbol & Quantity & Unit & PZT actuator & PZT sensor \\
\hline$L \times w \times t$ & Dimensions & $\mathrm{mm}$ & $46 \times 33.27 \times 0.25$ & $14 \times 7 \times 0.25$ \\
$d_{33}$ & Strain coefficient & $\mathrm{C} / \mathrm{N}$ & $7.41 \times 10^{-10}$ & $7.41 \times 10^{-10}$ \\
$d_{31}$ & Strain coefficient & $\mathrm{C} / \mathrm{N}$ & $-2.74 \times 10^{-10}$ & $-2.74 \times 10^{-10}$ \\
$\rho_{P}$ & PZT density & $\mathrm{kg} / \mathrm{m}^{3}$ & 7500 & 7500 \\
$E_{P}$ & Young's modulus & $\mathrm{N} / \mathrm{m}^{2}$ & $6.3 \times 10^{10}$ & $6.3 \times 10^{10}$ \\
\hline
\end{tabular}

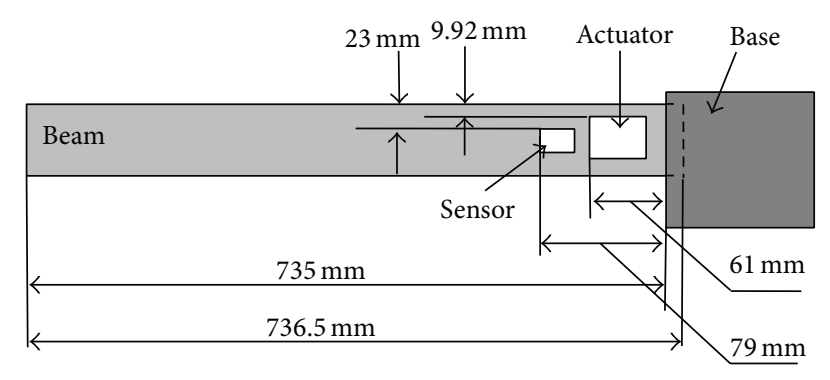

FIGURE 2: The physical position of the piezoelectric actuators and sensor.

of the model. The plant is represented in the form of the following general simplified dynamic equation:

$$
m \ddot{x}+c \dot{x}+k x=u
$$

where $m, c$, and $k$ are the coefficients relative with the system mass, damping, and stiffness, respectively, $x$ is the PZT sensor signal, and $u$ is PZT actuator signal.

\section{Design of Adaptive Robust Sliding Mode Controller and Stability Proof}

The design principle of the proposed adaptive robust sliding mode controller for vibration control is similar to that of the adaptive sliding mode controller for robot manipulator in [1, $6,31]$.

To assist control design, define $e=x_{r}-x$, where the subscript " $r$ " stands for the reference command. Define the sliding surface variable as

$$
s=\dot{e}+\lambda e=\dot{x}_{r}-\dot{x}+\lambda\left(x_{r}-x\right),
$$

where $\lambda$ is a positive number. $s=0$ represents the sliding surface, where control error converges to zero as $t \rightarrow \infty$.

By using a bang-bang robust compensator, the controller is proposed as

$$
u=\widehat{m}\left(\ddot{x}_{r}+\lambda\left(\dot{x}_{r}-\dot{x}\right)\right)+\widehat{c} \dot{x}+\widehat{k} x+a \operatorname{sgn}(s),
$$

where $\widehat{m}, \widehat{c}$, and $\widehat{k}$ are the initial estimations of the real values of parameters of $m, c$, and $k$, and these estimates will be adjusted online by the sliding mode based adaptive controller. The term $a \operatorname{sgn}(s)$ is the bang-bang robust compensator to ensure stability, where $a$ is a positive constant and the sgn function is defined as

$$
\begin{array}{ll}
\operatorname{sgn}(s)=+1 & \text { if } s>0, \\
\operatorname{sgn}(s)=-1 & \text { if } s<0 .
\end{array}
$$

With this feedback controller (3), the dynamic equation of the system (1) becomes

$$
m \ddot{x}+c \dot{x}+k x=\widehat{m}\left(\ddot{x}_{r}+\lambda\left(\dot{x}_{r}-\dot{x}\right)\right)+\widehat{c} \dot{x}+\widehat{k} x+a \cdot \operatorname{sgn}(s),
$$

which can be rewritten as

$$
\begin{aligned}
& \ddot{x}=\frac{1}{m}\left\{\widehat{m}\left[\ddot{x}_{r}+\lambda\left(\dot{x}_{r}-\dot{x}\right)\right]+a \cdot \operatorname{sgn}(s)\right. \\
&+\widehat{c} \dot{x}+\widehat{k} x-c \dot{x}-k x\}, \\
& \ddot{x}=\frac{1}{m}\left\{\widehat{m}\left[\ddot{x}_{r}+\lambda\left(\dot{x}_{r}-\dot{x}\right)\right]+m\left[\ddot{x}_{r}+\lambda\left(\dot{x}_{r}-\dot{x}\right)\right]\right. \\
& \quad-m\left[\ddot{x}_{r}+\lambda\left(\dot{x}_{r}-\dot{x}\right)\right]+a \cdot \operatorname{sgn}(s) \\
&\quad+\widehat{c} \dot{x}+\widehat{k} x-c \dot{x}-k x\}, \\
& \ddot{x}=\frac{1}{m}\left[\widetilde{m}\left(\ddot{x}_{r}+\lambda\left(\dot{x}_{r}-\dot{x}\right)\right)+\widetilde{c} \dot{x}+\widetilde{k} x+a \cdot \operatorname{sgn}(s)\right] \\
&+\ddot{x}_{r}+\lambda\left(\dot{x}_{r}-\dot{x}\right),
\end{aligned}
$$

where $\widetilde{m}=\widehat{m}-m, \widetilde{c}=\widehat{c}-c$ and $\widetilde{k}=\widehat{k}-k$.

Define the Lyapunov function candidate as

$$
v=\frac{1}{2} m s^{2}+\frac{1}{2} \widetilde{\varphi}^{T} \Gamma^{-1} \widetilde{\varphi},
$$

where $\tilde{\varphi}=\left[\begin{array}{lll}\widetilde{\varphi}_{1} & \widetilde{\varphi}_{2} & \widetilde{\varphi}_{3}\end{array}\right]^{T}=\left[\begin{array}{lll}\widetilde{m} & \widetilde{c} & \tilde{k}^{T}\end{array}\right]^{T}$ and $\Gamma=\left[\begin{array}{ccc}r_{1} & 0 & 0 \\ 0 & r_{2} & 0 \\ 0 & 0 & r_{3}\end{array}\right]$ $\left(r_{1}>0, r_{2}>0\right.$, and $\left.r_{3}>0\right)$ is a positive definite and diagonal matrix. Consider

$$
\begin{gathered}
\dot{v}=s m \dot{s}+\tilde{\varphi}^{T} \Gamma^{-1} \dot{\tilde{\varphi}} \\
\dot{v}=\operatorname{sm}\left(\ddot{x}_{r}-\ddot{x}+\lambda\left(\dot{x}_{r}-\dot{x}\right)\right)+\tilde{\varphi}^{T} \Gamma^{-1} \dot{\tilde{\varphi}} .
\end{gathered}
$$

Substituting (6) in (8) gives

$$
\begin{aligned}
\dot{v}=\operatorname{sm}\left\{\ddot{x}_{r}-\frac{1}{m}\left[\widetilde{m}_{x_{r}}+\lambda\left(\dot{x}_{r}-\dot{x}\right)\right.\right. \\
+\widetilde{c} \dot{x}+\widetilde{k} x+a \cdot \operatorname{sgn}(s)] \\
\left.-\left(\ddot{x}_{r}+\lambda\left(\dot{x}_{r}-\dot{x}\right)\right)+\lambda\left(\dot{x}_{r}-\dot{x}\right)\right\}+\widetilde{\varphi}^{T} \Gamma^{-1} \dot{\tilde{\varphi}}
\end{aligned}
$$




$$
\begin{gathered}
=-s\left\{\widetilde{m}\left[\ddot{x}_{r}+\lambda\left(\dot{x}_{r}-\dot{x}\right)\right]+\widetilde{c} \dot{x}+\widetilde{k} x\right. \\
+a \cdot \operatorname{sgn}(s)\}+\widetilde{\varphi}^{T} \Gamma^{-1} \dot{\tilde{\varphi}} .
\end{gathered}
$$

Define $Y=\left[\begin{array}{lll}y_{1} & y_{2} & y_{3}\end{array}\right]^{T}$, where $y_{1}=\ddot{x}_{r}+\lambda\left(\dot{x}_{r}-\dot{x}\right), y_{2}=\dot{x}$, and $y_{3}=x$.

We will obtain

$$
\widetilde{m}\left(\ddot{x}_{r}+\lambda\left(\dot{x}_{r}-\dot{x}\right)\right)+\widetilde{c} \dot{x}+\widetilde{k} x=Y^{T} \widetilde{\varphi} .
$$

Therefore, (9) is expressed as

$$
\begin{aligned}
\dot{v} & =-s\left(Y^{T} \widetilde{\varphi}+a \cdot \operatorname{sgn}(s)\right)+\widetilde{\varphi}^{T} \Gamma^{-1} \dot{\tilde{\varphi}} \\
& =-a \cdot s \cdot \operatorname{sgn}(s)-s Y^{T} \widetilde{\varphi}+\widetilde{\varphi}^{T} \Gamma^{-1} \dot{\tilde{\varphi}} \\
& =-a \cdot s \cdot \operatorname{sgn}(s)-\widetilde{\varphi}^{T}\left(Y s-\Gamma^{-1} \dot{\tilde{\varphi}}\right) .
\end{aligned}
$$

Notice that $-a \cdot s \cdot \operatorname{sgn}(s) \leq 0$.

The adaptation law will be designed as $Y s-\Gamma^{-1} \dot{\tilde{\varphi}}=0$. Therefore, $\dot{v}=-a \cdot s \cdot \operatorname{sgn}(s) \leq 0$, while $\dot{v}=0$ only when $s=0$. According to LaSalle's theorem, the control system is asymptotically stable in the sense of Lyapunov if the adaptation law satisfies $Y s-\Gamma^{-1} \dot{\tilde{\varphi}}=0$. In the sliding mode controller, the positive constant $a$, which is the amplitude of the robust compensator in control law (3), needs to be the upper bounding of the uncertainty of the system model and the external disturbance to ensure the stability. Due to the lack of the prior knowledge of the system, the upper bounding of the uncertainty and the disturbance may be difficult to be properly or precisely evaluated. In order to ensure the asymptotic stability of the system under all conditions, the amplitude of the robust compensator usually needs to be chosen large enough to compensate for the worst case of all possible uncertainty and external disturbances. Therefore, in the sliding mode controller, the transient performance and the steady state performance of the controller are usually sacrificed for the asymptotic stability of the control system. However, in the proposed adaptive controller, the adaptation law is designed to guarantee the asymptotic stability under uncertainty and disturbance. Therefore, there will be less constraint on the choice of the amplitude $a$ of the robust compensator in the proposed method. Conservatism of the robust controller would be dramatically reduced by introducing an adaptive scheme into the controller. The asymptotic stability of the proposed adaptive sliding mode controller is guaranteed with larger values of allowable uncertainty and disturbances. Both adaptive control part and sliding mode control part could compensate for the system uncertainties and external disturbances. The adaptation law can be derived as

$$
Y s-\Gamma^{-1} \dot{\tilde{\varphi}}=0 \Longrightarrow \dot{\tilde{\varphi}}=\Gamma Y s .
$$

Therefore,

$$
\begin{aligned}
\dot{\tilde{m}} & =\dot{\tilde{\varphi}}_{1}=\gamma_{1} y_{1} s=\gamma_{1}\left(\ddot{x}_{r}+\lambda\left(\dot{x}_{r}-\dot{x}\right)\right)(\dot{e}+\lambda e), \\
\dot{\tilde{c}} & =\dot{\tilde{\varphi}}_{2}=\gamma_{2} y_{2} s=\gamma_{2} \dot{x}(\dot{e}+\lambda e), \\
\dot{\widetilde{k}} & =\dot{\tilde{\varphi}}_{3}=\gamma_{3} y_{3} s=\gamma_{3} x(\dot{e}+\lambda e) .
\end{aligned}
$$

Notice that $\dot{\bar{m}}=\dot{\vec{m}}, \dot{\vec{c}}=\dot{\vec{c}}$, and $\dot{\hat{k}}=\dot{\tilde{k}}$.
The estimates will be updated according to the following equations:

$$
\begin{gathered}
\widehat{m}=\widehat{m}_{0}+\int \dot{\vec{m}} d t=\widehat{m}_{0}+\int \dot{\bar{m}} d t \\
=\widehat{m}_{0}+\int \gamma_{1}\left(\ddot{x}_{r}+\lambda\left(\dot{x}_{r}-\dot{x}\right)\right)(\dot{e}+\lambda e) d t, \\
\widehat{c}=\widehat{c}_{0}+\int \dot{\vec{c}} d t=\widehat{c}_{0}+\int \dot{\tilde{c}} d t=\widehat{c}_{0}+\int \gamma_{2} \dot{x}(\dot{e}+\lambda e) d t, \\
\widehat{k}=\widehat{k}_{0}+\int \dot{\hat{k}} d t=\widehat{k}_{0}+\int \dot{\tilde{k}} d t=\widehat{k}_{0}+\int \gamma_{3} x(\dot{e}+\lambda e) d t,
\end{gathered}
$$

where $\widehat{m}_{0}, \widehat{c}_{0}$, and $\widehat{k}_{0}$ are the initial values.

In vibration controls, the reference command is designed as

$$
x_{r}=0, \quad \dot{x}_{r}=0, \quad \ddot{x}_{r}=0 .
$$

With (15), the controller (3) can be simplified to

$$
u=(-\lambda \widehat{m}+\widehat{c}) \dot{x}+\widehat{k} x+a \cdot \operatorname{sgn}(s) .
$$

The shortcoming of the adaptive robust sliding mode controller (3) is that the bang-bang robust compensator will cause chattering in implementation. The chattering phenomenon is caused by the sign function term. The sign function switches the control action abruptly, especially near the equilibrium point. Chattering is not desirable, since it involves extremely high control activity and may excite high frequency dynamics of a structure.

\section{Design of a Smooth Adaptive Robust Sliding Mode Controller and Stability Proof}

In order to attenuate the chattering associated with the bangbang compensator, a hyperbolic tangent function is used to switch the control action instead of the sign function. The controller becomes

$$
u=\widehat{m}\left(\ddot{x}_{r}+\lambda\left(\dot{x}_{r}-\dot{x}\right)\right)+\widehat{c} \dot{x}+\widehat{k} x+a \tanh (\alpha s),
$$

where $a$ and $\alpha$ are positive numbers and tanh is the hyperbolic tangent function:

$$
\tanh (x)=\frac{e^{x}-e^{-x}}{e^{x}+e^{-x}} .
$$

The $a \tanh (\alpha s)$ is a robust compensator which provides a smooth control action. The hyperbolic function will switch the control action smoothly and avoid causing chattering. This robust compensator is continuously differentiable with respect to the control variable $s$. It generates a smooth control action. Compared with the commonly used bangbang or saturation robust controllers, the smooth robust controller has advantages in ensuring smooth control input and the stability of the closed-loop system [32]. A detailed comparative study is provided in Song and Mukherjee [1]. Normally, the larger the value of the parameter $\alpha$ is, the closer 
the $\tanh (\alpha s)$ function will mimic the $\operatorname{sign}(s)$ function. To avoid chattering, a relative smaller value of $\alpha$ can be chosen. When implementing the controller, the value of $\alpha$ is often experimentally determined.

To prove the stability of the smooth adaptive robust controller (17), define the Lyapunov function candidate as

$$
v=\frac{1}{2} m s^{2}+\frac{1}{2} \tilde{\varphi}^{T} \Gamma^{-1} \tilde{\varphi}
$$

Using the similar process shown in Section 3, we have

$$
\dot{v}=-a \cdot s \cdot \tanh (\alpha s)-\widetilde{\varphi}^{T}\left(Y s-\Gamma^{-1} \dot{\tilde{\varphi}}\right) .
$$

Please note that $\widehat{m}, \widehat{c}$, and $\widehat{k}$ are updated according to (14) to make $Y s-\Gamma^{-1} \dot{\tilde{\varphi}}=0$. Then, $\dot{v}=-a \cdot s \cdot \tanh (\alpha s) \leq 0$, while $\dot{v}=0$ only when $s=0$. Therefore, according to LaSalle's theorem, the closed-loop control system with the smooth adaptive robust controllers (17) is asymptotically stable in the sense of Lyapunov.

\section{Experiment Results}

5.1. Open Loop Testing of the Aluminum Beam. First, open loop testing is performed to find the dominant mode of the beam for active vibration control. The beam was subjected to a mechanical impact at its midlength for a multimode excitation. The power spectrum density (PSD) plots of the 30 -second time response of the multimode excitation were obtained. The corresponding frequencies of peaks in the PSD plots represent the modal frequencies of the flexible beam. To study the attenuation of vibration at each modal frequency, the PSD plots of the first 5 seconds and the last 5 seconds of the beam vibration are shown in Figure 3. The peaks in Figure 3 clearly reveal the modal frequencies of the flexible beam: the 1st mode at $1.64 \mathrm{~Hz}$, the 2 nd mode at $9.77 \mathrm{~Hz}$, and the 3rd mode at $26.85 \mathrm{~Hz}$. A comparison of PSD plots of the vibration data for the first 5 seconds and the last 5 seconds clearly shows the vibration attenuation at each modal frequency. The $\mathrm{dB}$ value and $\mathrm{dB}$ drop of the first three modes are shown in Table 3. From Figure 3 and Table 3, it can be seen that the $\mathrm{dB}$ levels of the second and third modes at the first five-second and last five-second periods are significantly lower than that of the 1st mode. The $\mathrm{dB}$ drop value of the first three modes shows that the second and third modes attenuated much faster than the first mode. Therefore, it can be concluded that the first modal frequency, $1.64 \mathrm{~Hz}$, is the dominant one in the vibration and the first mode should be the target mode in the active vibration control. To target at this mode, the vibration control is designed based on the simplified plant (1), which includes only the dynamics of the first mode.

\subsection{Experimental Results for the Adaptive Robust Sliding} Mode Controller. To verify the effectiveness of the adaptive robust sliding mode control (3) and the smooth adaptive sliding mode control (17), experiments were conducted on the aluminum flexible beam. In the experiment, $s=\dot{e}+$ $\lambda e=\dot{e}+0.05 e$ and $r_{1}=r_{2}=r_{3}=0.1$ for the diagonal
TABLE 3: PSD drop comparison of first 5 seconds and last 5 seconds.

\begin{tabular}{lccc}
\hline & Mode 1 & Mode 2 & Mode 3 \\
\hline $\mathrm{dB}$ level of the first 5 seconds & 16.73 & 5.98 & -14.36 \\
$\mathrm{~dB}$ level of the last 5 seconds & 2.91 & -17.23 & -35.19 \\
$\mathrm{~dB}$ drop & 13.82 & 23.21 & 20.83 \\
\hline
\end{tabular}

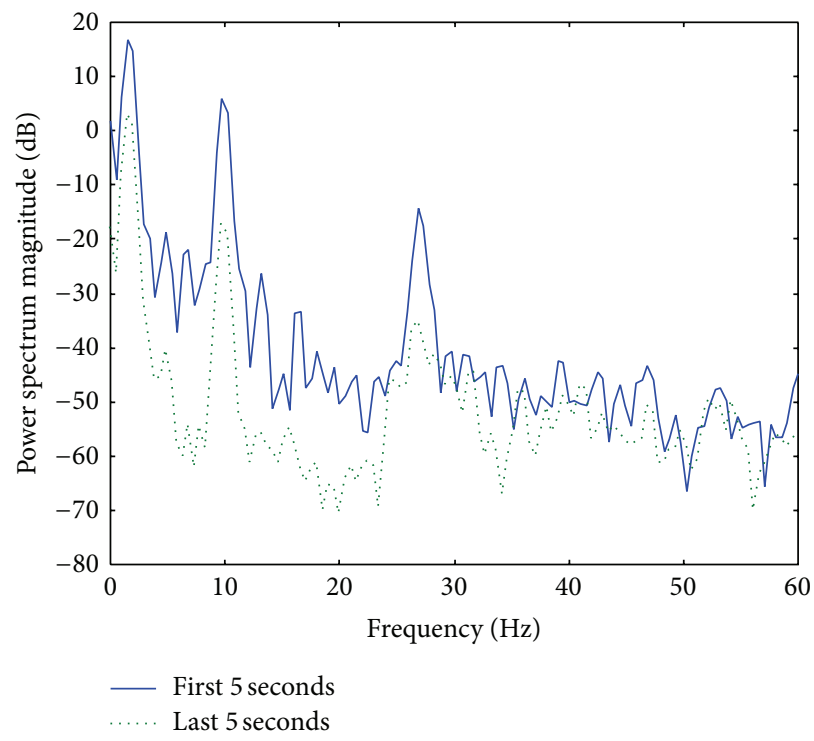

FIgure 3: Comparison of PSD plots of first 5 seconds and last 5 seconds for time response of multimode excitation of the flexible beam.

matrix $\Gamma$. In each test, the beam was excited by a sinusoidal signal at its first modal frequency combined with a low power white noise for the initial 5 seconds. The amplitude of the sinusoidal signal was $0.75 \mathrm{~V}$. The variance of the white noise was 0.0497 . The sinusoidal signal and the white noise were summed in the Simulink software and downloaded into dSPACE DS1102 Floating-Point Controller Board for realtime implementation. The output of the dSPACE controller board was amplified by an amplifier for capacitive load before exciting the PZT actuators. After the initial 5 seconds, first mode excitation was stopped and the active vibration control started to suppress the induced vibration. A free vibration test was conducted for the purpose of comparison. In the free vibration test, no control signal was implemented after the initial 5-second excitation.

The upper diagram of Figure 4 is the time response comparison of free vibration with that of the active control (with the sign function). From the experimental results, the vibration is suppressed in about 8 oscillation cycles (about 5 seconds). However, there is small-magnitude chattering in the steady state, which may cause instability when a certain level of disturbance exists. The lower diagram of Figure 4 is the time response comparison of free vibration with that of the active control (with the tanh function). It can be seen that the robust sliding mode control can suppress the vibration in about 8 oscillation cycles without the chattering in the steady state. Experimental results show that the chattering can be 


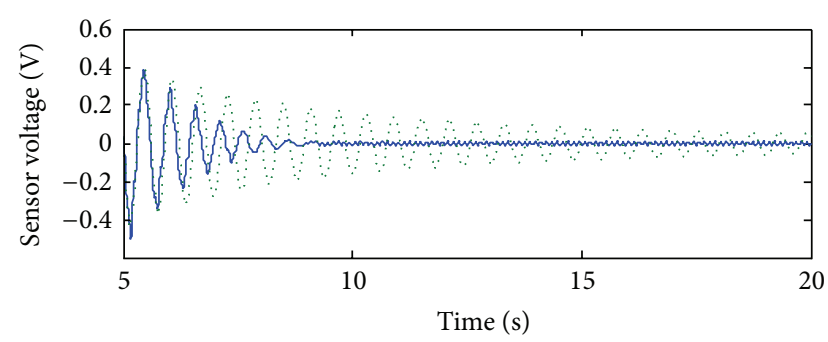

_ Sliding-mode control with sign function ..... Free vibration

(a)

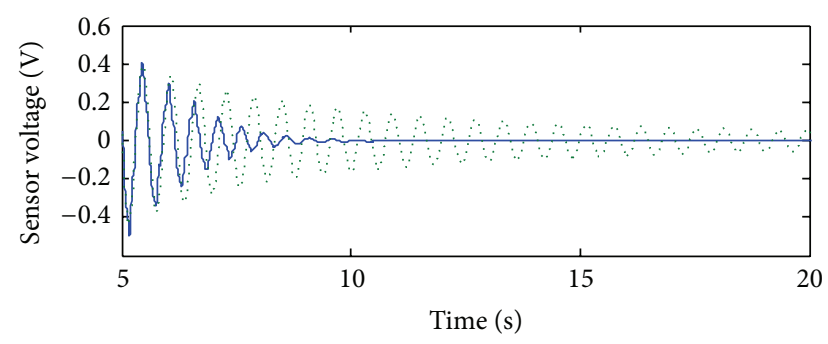

_ Sliding-mode control with tanh function Free vibration

(b)

FIGURE 4: The time response comparisons of the adaptive sliding mode controller (with the sign function) with the adaptive robust sliding mode controller (with tanh function).

eliminated by implementing the hyperbolic tangent function (tanh function) as the switching function instead of the sign function.

The upper diagram of Figure 5 is the control action with the bang-bang robust compensator. Compared with the time response in Figure 4, it can be seen that the chattering happens when the control action switches abruptly between its positive limit and negative limit when the system is already in the steady state (after 10 seconds). The lower diagram of Figure 5 is the control action with the smooth robust compensator. From the diagram, it can be seen that the control signal switches smoothly. Therefore, the chattering in the output signal is eliminated. Figure 6 shows the time history of the parameters' updating process. Furthermore, it is also worthwhile to note that no higher modes have been excited, even though the plant is actually a multimode system. This justifies that the primary vibration control target is the first mode.

5.3. Experimental Results for the Adaptive Robust Sliding Mode Control with Mass Uncertainty. To verify the robustness of the proposed adaptive robust controller, the plant is changed by adding mass on the beam. Four 6-gram adhesive masses were attached to both sides of the beam, with two at the remote tip and the other two at the midlength of the beam. The mass of the aluminum beam is increased from 105.20 gram to 129.20 gram. The first modal frequency of the original beam is $1.67 \mathrm{~Hz}$, whereas the first modal frequency of the

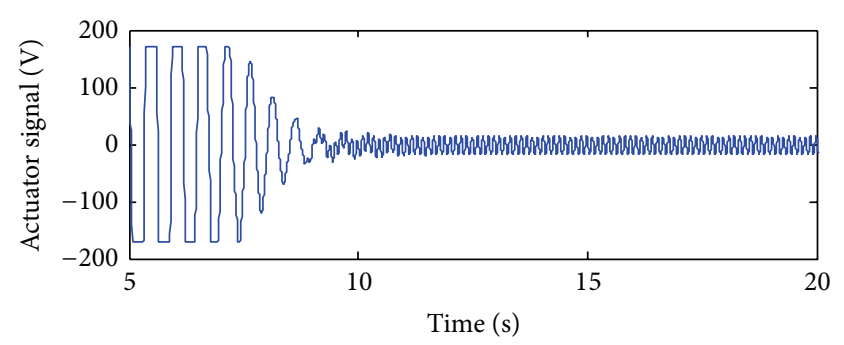

— Sliding-mode control with sign function

(a)

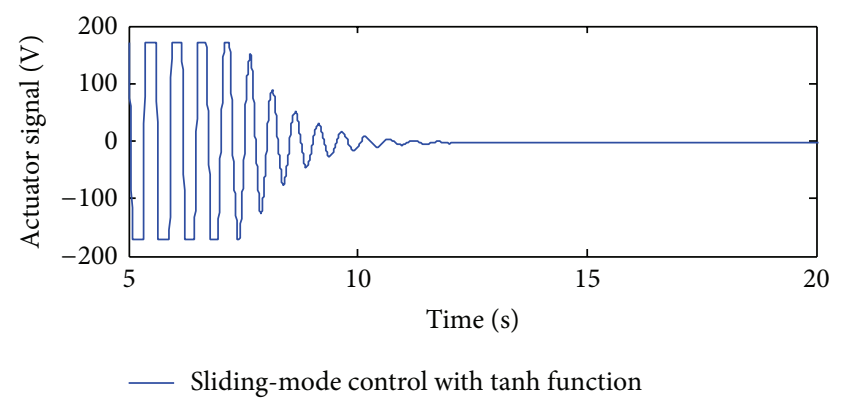

(b)

FIGURE 5: The control signal comparisons of the adaptive sliding mode controller (with the sign function) with the adaptive robust sliding mode controller (with tanh function).
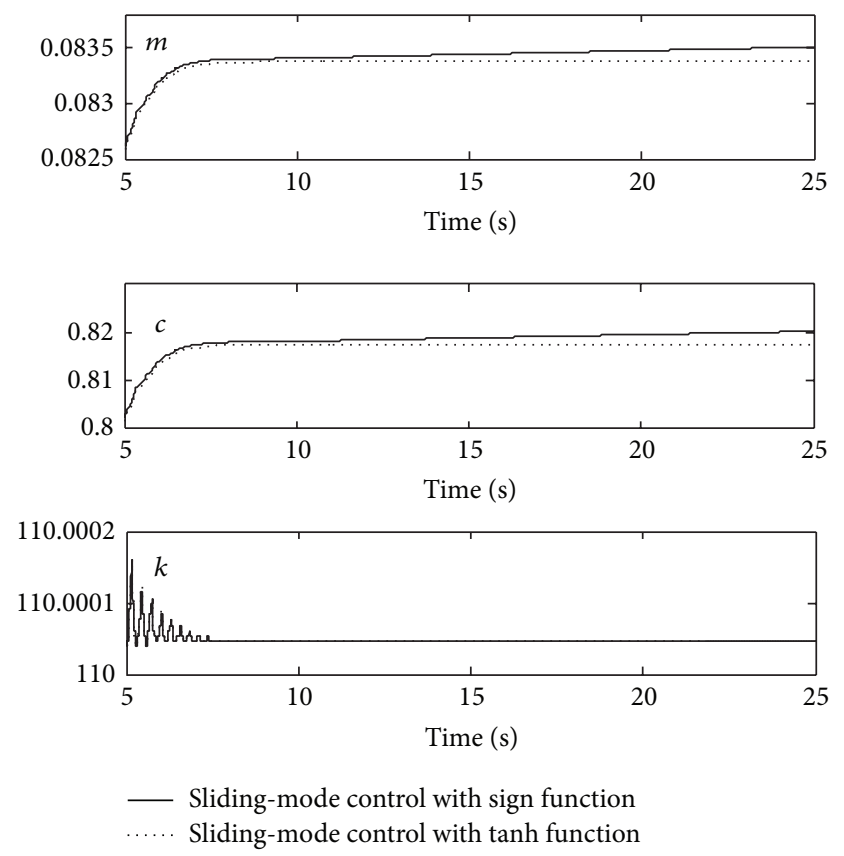

FIGURE 6: The parameters' updating time history.

beam with added mass has been decreased to $1.457 \mathrm{~Hz}$. The increasing of the mass has caused the decreasing of the natural frequency of the beam. Therefore, in this experiment, the frequency of the sinusoidal excitation signal has been changed to $1.457 \mathrm{~Hz}$ to ensure excitation of the beam. The same controller is used for the vibration control of the beam with added mass. From the experimental results (Figure 7), 


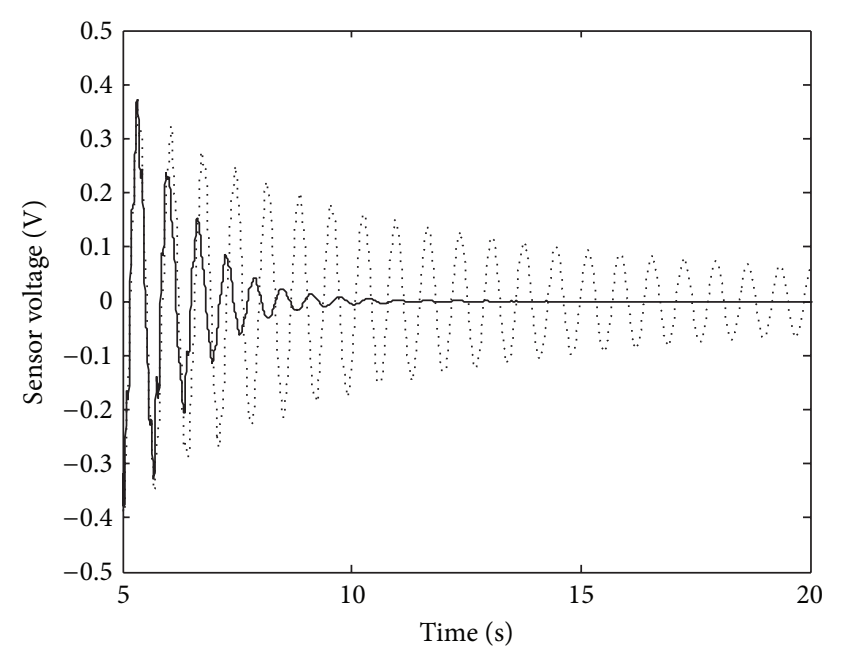

_ Sliding-mode control with tanh function ...... Free vibration

FIGURE 7: The time response comparisons of the adaptive sliding mode controller with tanh function for the flexible beam with 24gram mass added.

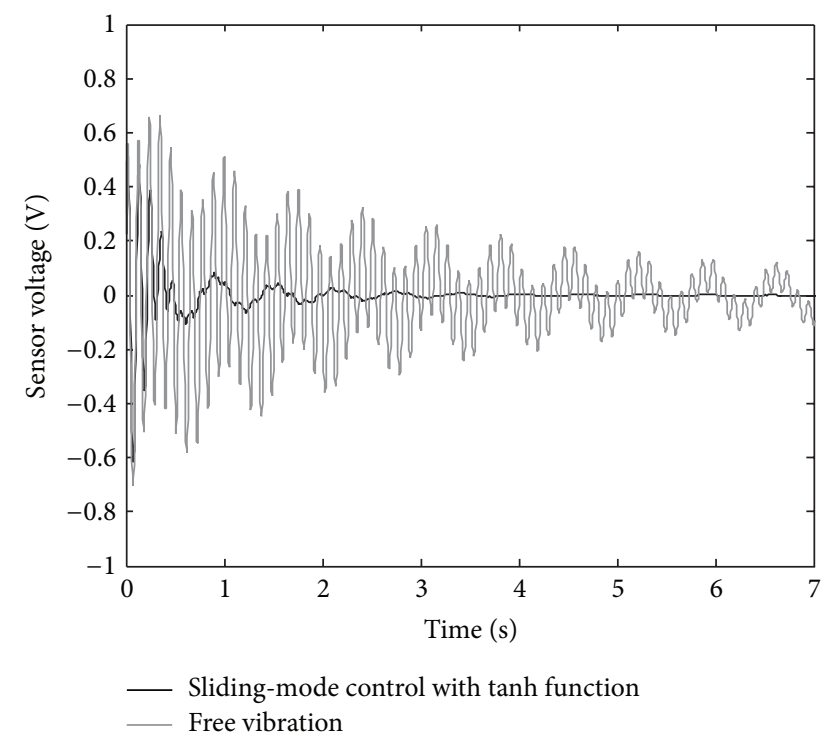

Figure 8: The time response of comparison of the adaptive sliding mode controller with tanh function for the flexible beam with multimode excitation.

it can be seen that the adaptive robust sliding mode can effectively suppress the vibration of the beam after its physical property has been changed. This demonstrates the robustness of the proposed controllers.

\subsection{Experimental Results for the Adaptive Robust Sliding Mode} Control with Multimode Excitation. To test its robustness to higher order dynamics and disturbances, multimodal excitation of the flexible beam with mass added is conducted. Figure 8 shows the comparison of time response of multimodal excitation with that of the free vibration. Experimental results clearly show that the proposed method is still effective in multimodal vibration control and this further demonstrates the robustness of the proposed controller.

\section{Conclusion}

In this paper, an experimental study of the robust adaptive sliding mode control was conducted on a flexible beam with piezoceramic actuators and sensor for vibration control purpose. Two different schemes of adaptive sliding mode control have been comparatively studied during the experiment. The adaptive robust sliding mode control with bangbang action could suppress the induced vibration of a flexible beam in a quick fashion. However, it causes chattering during steady state. To eliminate the chattering, a hyperbolic tangent function is used to replace the sign function in the robust control action. Both adaptive robust sliding mode controllers are designed based on the Lyapunov direct method. In the proposed adaptive sliding mode controller, there will be less constraint on the choice of the amplitude of the robust compensator. The conservatism of the control system has been reduced by introducing the adaptive scheme into the sliding mode control. The asymptotic stability of the adaptive robust sliding mode control is proved by using Lyapunov direct method in the paper. The experimental results show that the proposed adaptive robust sliding mode control with the smooth robust compensator eliminates the chattering while keeping the advantages of adaptive robust sliding mode control. Also the proposed methods are robust to the variance of the plant parameters, as demonstrated experimentally.

\section{Conflict of Interests}

The authors declare that there is no conflict of interests regarding the publication of this paper.

\section{Acknowledgment}

This research was partially supported by Grant no. 51278387, Grant no. 51278084, and Grant no. 51121005 (Science Fund for Creative Research Groups) from the National Science Foundation of China (NSFC).

\section{References}

[1] G. Song and R. Mukherjee, "A comparative study of conventional nonsmooth time-invariant and smooth time-varying robust compensators," IEEE Transactions on Control Systems Technology, vol. 6, no. 4, pp. 571-576, 1998.

[2] J.-H. Park, K.-W. Kim, and H.-H. Yoo, “Two-stage sliding mode controller for vibration suppression of a flexible pointing system," Proceedings of the Institution of Mechanical Engineers, Part C: Journal of Mechanical Engineering Science, vol. 215, no. 2, pp. 155-166, 2001.

[3] X. Yu, Z. Man, and B. Wu, "Design of fuzzy sliding-mode control systems," Fuzzy Sets and Systems, vol. 95, no. 3, pp. 295-306, 1998. 
[4] B. Yao and M. Tomizuka, "Smooth robust adaptive sliding mode control of manipulators with guaranteed transient performance," in Proceedings of the American Control Conference, vol. 1, pp. 1176-1180, July 1994.

[5] J. Wang, A. B. Rad, and P. T. Chan, "Indirect adaptive fuzzy sliding mode control: part I: fuzzy switching," Fuzzy Sets and Systems, vol. 122, no. 1, pp. 21-30, 2001.

[6] G. Song, R. W. Longman, and R. Mukherjee, "Integrated sliding-mode adaptive-robust control," IEE Proceedings: Control Theory and Applications, vol. 146, no. 4, pp. 341-347, 1999.

[7] G. Song, R. W. Longman, and L. Cai, "Integrated adaptiverobust control of robot manipulators," Journal of Robotic Systems, vol. 15, no. 12, pp. 699-712, 1998.

[8] R.-J. Wai, "Adaptive sliding-mode control for induction servomotor drive," IEE Proceedings: Electric Power Applications, vol. 147, no. 6, pp. 553-562, 2000.

[9] S.-C. Lin and Y.-Y. Chen, "Design of adaptive fuzzy sliding mode for nonlinear system control," in Proceedings of the 3rd IEEE Conference on Fuzzy Systems, pp. 35-39, June 1994.

[10] J. Fei and H. Ding, "Adaptive vibration control of microelectromechanical systems triaxial gyroscope using radial basis function sliding mode technique," Proceedings of the Institution of Mechanical Engineers, Part I: Journal of Systems and Control Engineering, vol. 227, no. 2, pp. 264-269, 2013.

[11] B. L. Zhang and Q. L. Han, "Robust sliding mode $H_{\infty}$ control using time-varying delayed states for offshore steel jacket platforms," in Proceedings of the IEEE International Symposium on Industrial Electronics (ISIE '13), pp. 1-6, IEEE, 2013.

[12] H. Zhang, G. Dong, M. Zhou, C. Song, Y. Huang, and K. Du, "A new variable structure sliding mode control strategy for FTS in diamond-cutting microstructured surfaces," The International Journal of Advanced Manufacturing Technology, vol. 65, no. 5-8, pp. 1177-1184, 2013.

[13] H. Li, J. Yu, C. Hilton, and H. Liu, "Adaptive sliding mode control for nonlinear active suspension vehicle systems using TS fuzzy approach," IEEE Transactions on Industrial Electronics, vol. 60 , no. 8, 2013.

[14] J. L. Fanson and T. K. Caughey, "Positive position feedback control for large space structures," AIAA Journal, vol. 28, no. 4, pp. 717-724, 1990.

[15] G. Song, P. Qiao, V. Sethi, and A. Prasad, "Active vibration control of a smart pultruded fiber-reinforced polymer I-beam," in International Symposium on Smart Structures and Materials, vol. 4696 of Proceedings of SPIE, pp. 197-208, March 2002.

[16] P. Mayhan and G. Washington, "Fuzzy model reference learning control: a new control paradigm for smart structures," Smart Materials and Structures, vol. 7, no. 6, pp. 874-884, 1998.

[17] V. Bottega, A. Molter, J. S. O. Fonseca, and R. Pergher, "Vibration control of manipulators with flexible nonprismatic links using piezoelectric actuators and sensors," Mathematical Problems in Engineering, vol. 2009, Article ID 727385, 16 pages, 2009.

[18] A. Molter, V. Bottega, O. A. A. Da Silveira, and J. S. O. Fonseca, "Simultaneous piezoelectric actuator and sensor placement optimization and control design of manipulators with flexible links using SDRE method," Mathematical Problems in Engineering, vol. 2010, Article ID 362437, 23 pages, 2010.

[19] M. Ahmadian and K. M. Jeric, "On the application of shunted piezoceramics for increasing acoustic transmission loss in structures," Journal of Sound and Vibration, vol. 243, no. 2, pp. 347-359, 2001.
[20] J. Fei, Y. Fang, and C. Yan, “The comparative study of vibration control of flexible structure using smart materials," Mathematical Problems in Engineering, vol. 2010, Article ID 768256, 13 pages, 2010.

[21] S.-B. Choi, Y.-K. Park, and C.-C. Cheong, "Active vibration control of hybrid smart structures featuring piezoelectric films and electrorheological fluids," in The International Society for Optical Engineering, vol. 2717 of Proceedings of the SPIE, pp. 544-552, February 1996.

[22] A. K. Jha and D. J. Inman, "Sliding mode control of a Gossamer structure using smart materials," Journal of Vibration and Control, vol. 10, no. 8, pp. 1199-1220, 2004.

[23] S.-B. Choi and M.-S. Kim, "New discrete-time, fuzzy-slidingmode control with application to smart structures," Journal of Guidance, Control, and Dynamics, vol. 20, no. 5, pp. 857-864, 1997.

[24] S. Bashash and N. Jalili, "Robust multiple frequency trajectory tracking control of piezoelectrically driven micro/nanopositioning systems," IEEE Transactions on Control Systems Technology, vol. 15, no. 5, pp. 867-878, 2007.

[25] H. C. Liaw, B. Shirinzadeh, and J. Smith, "Sliding-mode enhanced adaptive motion tracking control of piezoelectric actuation systems for micro/nano manipulation," IEEE Transactions on Control Systems Technology, vol. 16, no. 4, pp. 826-833, 2008.

[26] Y. Li and Q. Xu, "Adaptive sliding mode control with perturbation estimation and PID sliding surface for motion tracking of a piezo-driven micromanipulator," IEEE Transactions on Control Systems Technology, vol. 18, no. 4, pp. 798-810, 2010.

[27] J. Hu and D. Zhu, "Vibration control of smart structure using sliding mode control with observer," Journal of Computers, vol. 7, no. 2, pp. 411-418, 2012.

[28] L. Zhang, Z. Zhang, Z. Long, and A. Hao, "Sliding mode control with auto-tuning law for Maglev System," Engineering, vol. 2, no. 2, pp. 107-112, 2010.

[29] T. Rittenschober and K. Schlacher, "Observer-based self sensing actuation of piezoelastic structures for robust vibration control," Automatica, vol. 48, no. 6, pp. 1123-1131, 2012.

[30] X. Bu, L. Ye, Z. Su, and C. Wang, "Active control of a flexible smart beam using a system identification technique based on ARMAX," Smart Materials and Structures, vol. 12, no. 5, pp. 845$850,2003$.

[31] R. D. Robinett, C. R. Dohrmann, G. R. Eisler et al., Flexible Robot Dynamics and Controls, Kluwer Academic/Plenum Publishers, New York, NY, USA, 2002.

[32] L. Cai and G. Song, "Joint stick-slip friction compensation of robot manipulators by using smooth robust controllers," Journal of Robotic Systems, vol. 11, no. 6, pp. 451-470, 1994. 


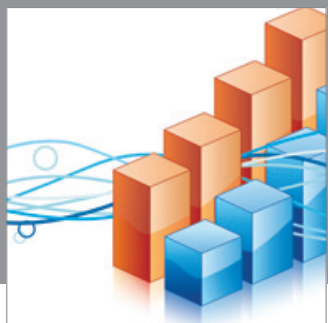

Advances in

Operations Research

mansans

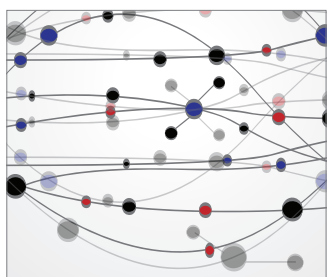

The Scientific World Journal
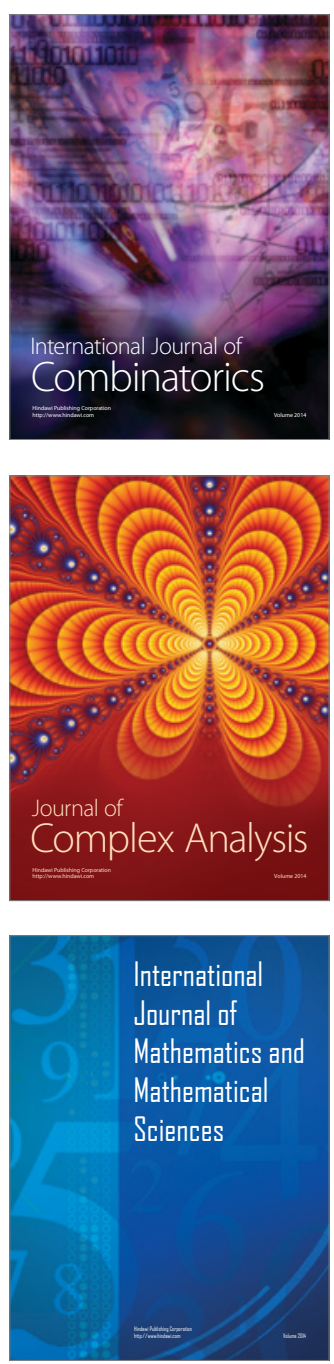
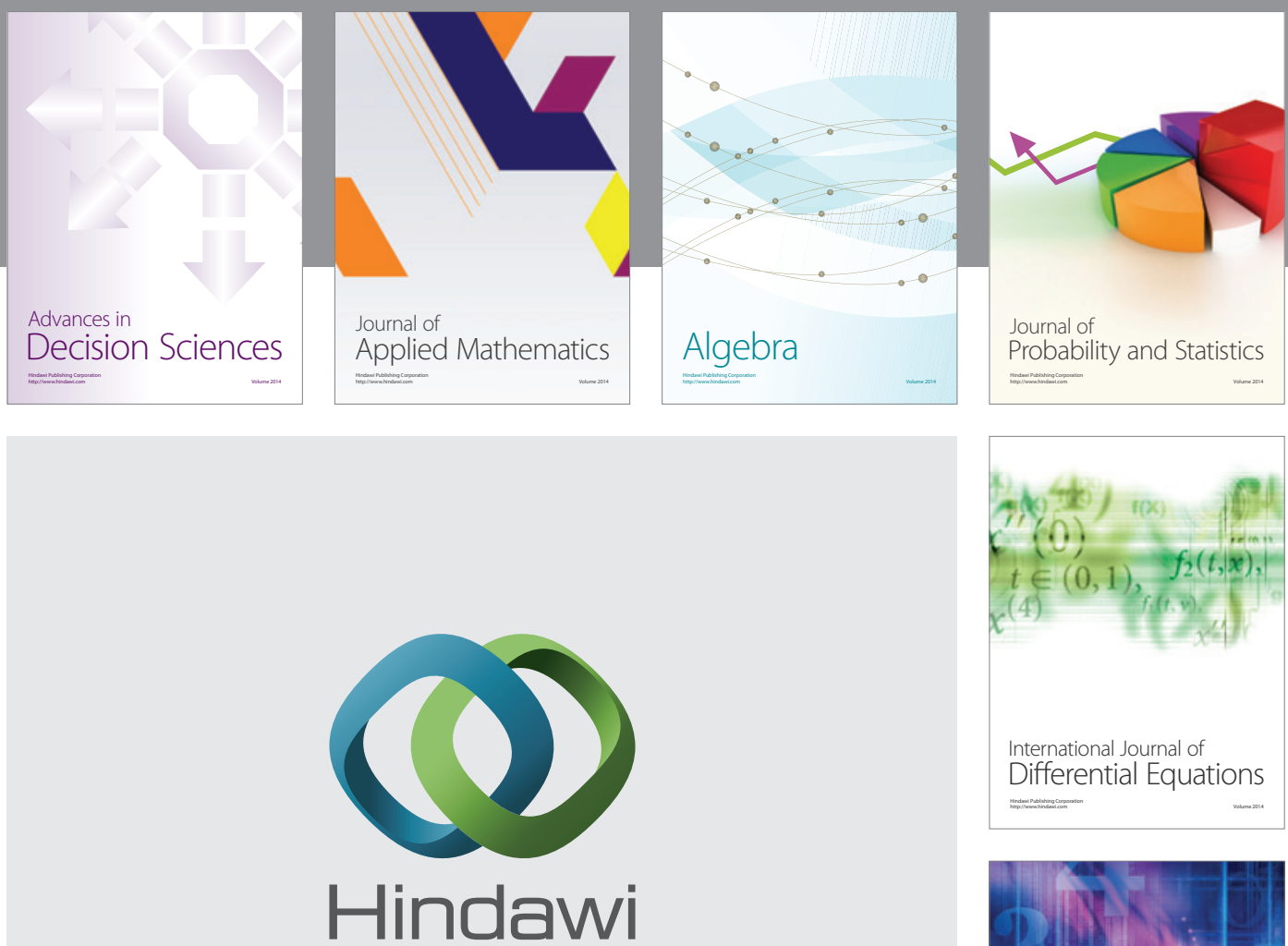

Submit your manuscripts at http://www.hindawi.com
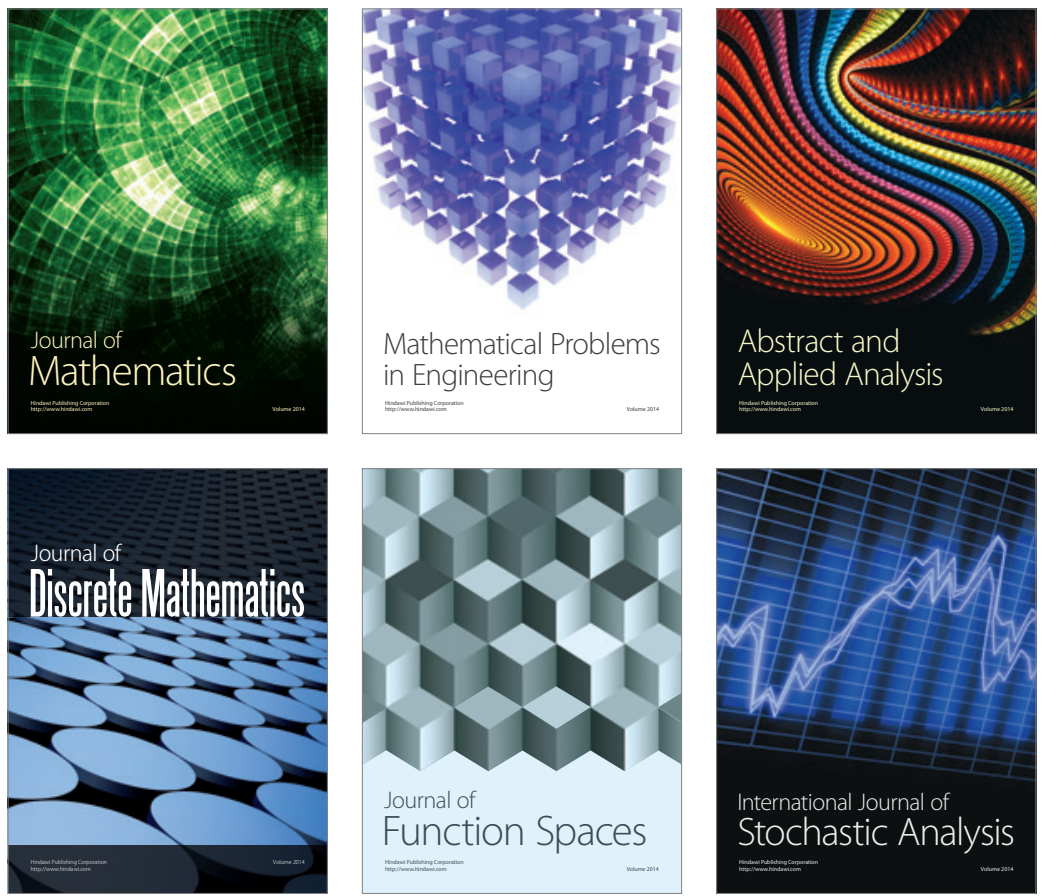

Journal of

Function Spaces

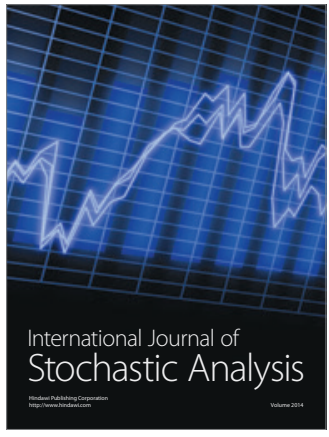

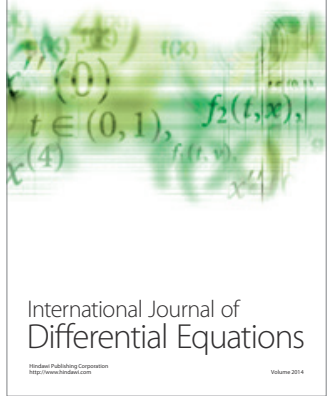
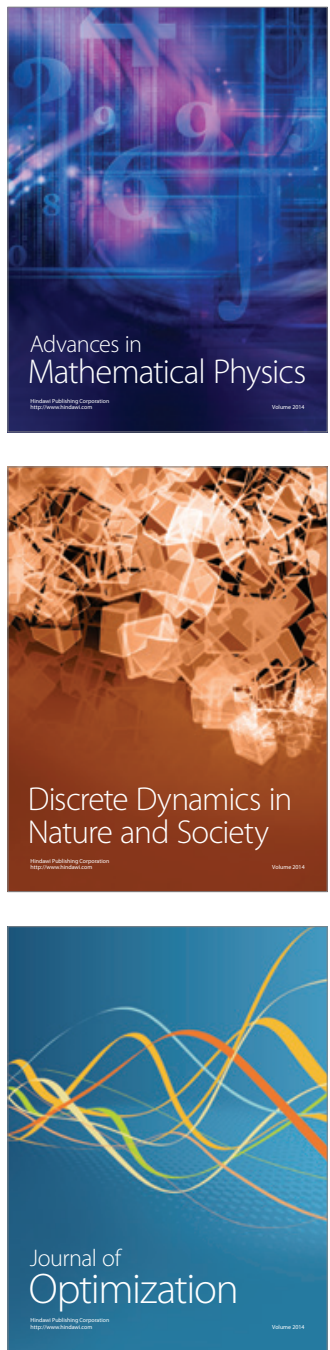